\title{
La gestación por sustitución como una forma de mercantilización del cuerpo femenino
}

\author{
Marta Szygendowska*
}

\begin{abstract}
RESUMEN
El presente trabajo efectúa una revisión y análisis acerca del contenido de la práctica de gestación por sustitución, poniendo el énfasis en cómo el desarrollo de sus formas, funciones y limitaciones se ha configurado a base de un contexto de dominación patriarcal y de expansión del capitalismo: modo de acumulación que ha comercializado las capacidades reproductivas de la mujer, instrumentalizando su cuerpo y tensionando el respeto del ejercicio de sus derechos individuales. Para lograr lo anterior, se realizará una explicación crítica acerca del contenido de la institución, de sus fines y modalidades, atendiendo la actual discusión teórica dentro del movimiento feminista.
\end{abstract}

Gestación por sustitución; feminismo; derechos de las mujeres

\section{Surrogacy as a form of commercialisation of the female body}

\begin{abstract}
This paper analyses and reviews a surrogacy practice, with the emphasis on how the development of its forms, functions and limitations have been configured on a base of patriarchal domination context and process of expansion of capitalism. The system of the accululation of capital commercializes the reproductive capacities of women, instrumentalizing their body and makes difficult to respect an excercise of their individual rights. To achieve the above, a critical explanation will be made about the content of the institution, its purposes and modalities, attending the current theorical discussion within the feminist movement
\end{abstract}

Surrogacy; feminism; women's rights

* Licenciada en Derecho, Universidad de Cardinal Wyszynski, Varsovia, Polonia. Máster en Estudios Internacionales y de la Unión Europea, Universidad de Valencia, España. Doctora en Derecho, mención Derechos Humanos, Paz y Desarrollo Sostenible, Universidad de Valencia, España. Profesora de la Facultad de Ciencias Jurídicas, Universidad de Antofagasta. ORCID: https://orcid.org/0000-0002-6385-9520. Correo electrónico: marta.szygendowska@uantof.cl

Artículo recibido el 4.3.20 y aceptado para su publicación el 18.6.20. 


\section{INTRODUCCIÓN}

$\mathrm{L}$ a mercantilización del cuerpo siempre ha sido vinculada a los debates relativos al tráfico de personas con fines de explotación sexual, tráfico de órganos humanos u otra forma de la llamada esclavitud moderna que involucra la venta del cuerpo. En el caso de la gestación por sustitución ${ }^{1}$, esta ha sido considerada por la gran parte de la doctrina como una forma de explotación reproductiva de mujeres ${ }^{2}$. Dicha institución ha ganado el protagonismo en los debates desarrollados en el área internacional, debido a que cada vez más personas infértiles de los países occidentales optan por esta solución para tener hijos. La discusión se centra en la tensión entre los derechos individuales de la gestante y la expansión del mercado que sobrepasa los límites morales y le pone precio a la vida. Los que defienden esta vía para resolver su problema y satisfacer su deseo de ser padre o madre, se apoyan en el derecho a formar una familia y respetar la decisión libre de la mujer que se decide a gestar un feto para otras personas ${ }^{3}$. Asimismo, los que abogan por la prohibición absoluta de esta práctica sostienen que esta conlleva a mercantilizar y cosificar el cuerpo de la mujer ${ }^{4}$.

En todo caso, la gestación por sustitución se ha ido convirtiendo en una industria desde los años ochenta y el negocio creado por las empresas intermediarias se ha expandido a lo largo del mundo, generando actualmente una ganancia de 2,3 mil millones de dólares al año ${ }^{5}$. Las agencias de gestación por sustitución que en realidad son empresas intermediarias que actúan entre los comitentes dispuestos a pagar una alta suma de dinero por tener un bebé, y la gestante que generalmente es proveniente de un entorno vulnerable $^{6}$, desarrollan su negocio en los países en vía de desarrollo. Así, se garantizan un coste reducido del servicio y la mayor ganancia. Debido a que en gran parte de los países occidentales la gestación por sustitución es una práctica que está prohibida, las agencias difunden su publicidad que abarrota internet. La oferta está dirigida a los comitentes provenientes de estos, ofreciéndoles precios más bajos por el servicio efectuado en los países empobrecidos.

En este sentido, la figura de la gestación por sustitución constituye una forma de mercantilización del cuerpo femenino, explotando a las mujeres vulnerables procedentes de los países en vía de desarrollo, generando una industria que debería estar prohibida.

\footnotetext{
${ }^{1}$ Denominada también como maternidad subrogada, gestación subrogada, vientres de alquiler, maternidad por encargo, alquiler de útero. Existen diferentes modalidades de esta práctica, por eso la terminología difiere.

2 Véase Albert, 2017.

${ }^{3}$ Eleonora Lamm mantiene que “(...) existe de forma básica y fundamental un derecho de las gestantes a servirse libremente de su cuerpo y a tomar decisiones al respecto”. LAMM, 2017, p. 539.

${ }^{4}$ No Somos Vasijas, s/f.

${ }^{5}$ Cottingham, 2017, p. 17.

${ }^{6}$ Ibid.
} 
En efecto, el objetivo de este trabajo es analizar la gestación por sustitución como una práctica que cosifica el cuerpo de la mujer y lo convierte en un objeto de consumo y explotación reproductiva.

\section{Gestación POR Sustitución: Antecedentes}

La gestación por sustitución consiste en la aceptación que hace una mujer para "portar en su vientre un niño por encargo de otra persona o de una pareja, con el compromiso de, una vez llevado a término el embarazo, entregar el recién nacido al comitente o comitentes, renunciando aquella a la filiación que pudiera corresponderle sobre el hijo así gestado"7. Aunque en la doctrina no existe un consenso acerca de si constituye una modalidad de reproducción asistida ${ }^{8}$, los partidarios de la regulación permisiva la consideran una de sus formas ${ }^{9}$. No obstante, si bien en el procedimiento se recurre a las técnicas de reproducción asistida, este se rige por un contrato cuyo objeto consiste en que la gestante entregue al recién nacido a los comitentes a cambio de un precio. Por tanto, es difícil considerar la gestación por sustitución como una forma de reproducción asistida ${ }^{10}$. Para los partidarios parece confortable catalogarla como tal, pero no hay que olvidar que lo que realmente se suele ocultar es un hecho incómodo en torno a que la capacidad reproductiva de la mujer se convierte en un negocio. Las personas que no pueden tener hijos recurren a esta práctica para satisfacer sus deseos de ser padres, encontrándose en el mercado con una inmensa cantidad de empresas que, por un precio establecido, llevan a cabo todo el procedimiento. De este modo, los que cuentan con recursos económicos pueden acceder a un servicio en el que la vida humana se reduce a un producto.

Los primeros casos de gestación por sustitución se hicieron públicos en Estados Unidos en la década de los años setenta donde se reconoció el primer acuerdo entre los comitentes y la gestante ${ }^{11}$, dando paso a este tipo de contratos cuyo número llegó a los 600 entre 1976 y $1988^{12}$. Sin embargo, no fue hasta 1986 cuando el tema generó una gran polémica a raíz del famoso caso Baby $M$, cuando la Corte Suprema estadounidense por primera vez tuvo que fallar acerca de la custodia de una bebé nacida de un contrato de gestación por sustitución, concediéndosela finalmente a los comitentes. A partir de entonces, el mercado de gestación por sustitución empezó a crecer en algunos estados de Estados Unidos donde se determinó legislar acerca de la cuestión a partir de los años noventa. Al mismo tiempo, el mercado de subrogación se expandió a los países en

${ }^{7}$ SÁnCHEZ 2010, p. 8. Para ver todas las formas de la gestación por sustitución, véase la clasificación de Capella, 2015, pp. 23-27.

${ }^{8}$ LARA, 2018.

9 Véase LAMm, 2017, p. 24.

${ }^{10}$ En el mismo sentido, véase RAYMOND, p. 51.

11 Sreenivas y Campo-Engelstein, 2010, p. 3.

12 Lehman y Phelps, 2005, pp. 408-16. 
vía de desarrollo, como India o Ucrania, debido a los bajos costos relacionados con la ejecución del contrato ${ }^{13}$.

Respecto de la regulación legal que hacen los países acerca del fenómeno, es posible evidenciar que, en algunos, tal práctica se encuentra prohibida en forma absoluta (como, por ejemplo, en Francia, Alemania, España, Italia). Otros optan por la regulación permisiva cuando es altruista (por ejemplo, Reino Unido, Canadá, Grecia ${ }^{14}$ ), mientras en países como Ucrania, Rusia, algunos estados de Estados Unidos, como el estado de California o el estado de Sinaloa en México ${ }^{15}$, domina la admisión amplia. En el caso de algunos países asiáticos que han sido los destinos más concurridos por las parejas extranjeras europeas, debido a los bajos costes del servicio (comparando con, por ejemplo, Estados Unidos), en los últimos años han prohibido la gestación por sustitución comercial. Este es el caso de Tailandia, que a raíz del caso Baby Gammy ${ }^{16}$ aprobó en el 2015 la ley que prohíbe expresamente la gestación por sustitución, basándose en la "protección de los niños subrogados y represión de la trata de personas"17. Por su parte, la Corte Suprema de Nepal prohibió la gestación por sustitución en una decisión dictada en el 2015, con excepción de las parejas nepalesas casadas ${ }^{18}$. En el caso de India, la ley que regula la gestación subrogada del año 2019 prohíbe la gestación comercial, subrayando la urgencia de la eliminación de esta práctica que había sido permitida hasta la fecha en comento, ya que "se han reportado casos de prácticas poco éticas, explotación de las madres sustitutas, abandono de niños nacidos de subrogación e importación de embriones humanos y gametos" 19 .

En cuanto a los países donde se ha planteado legislar acerca del tema, en Chile, el 2017 se presentó un proyecto de ley que regula la gestación por subrogación o gestación subrogada como mecanismo de reproducción asistida ${ }^{20}$. El objetivo del proyecto es establecer una regulación jurídica permisiva de la práctica de gestación por sustitución de carácter altruista, debido a la disminución, en los últimos años, de la tasa de natalidad en el país.

Por su parte, dentro de la Unión Europa, aunque no hay consenso de los Estados miembros acerca del tema en comento y los países han tomado diferentes posiciones en cuanto a la figura de la gestación por sustitución, la postura del Parlamento Europeo es

${ }^{13}$ Mohapatra, 2012, p. 413.

${ }^{14} \mathrm{La}$ ley permite ser comitentes a los ciudadanos griegos o comunitarios, siendo parejas heterosexuales o personas solteras.

15 Ávila, 2017, pp. 328-338.

${ }^{16}$ Es el caso de una pareja australiana que contrató a una mujer tailandesa como gestante. La mujer estaba embarazada de gemelos, uno de ellos con síndrome de Down. Después de haber rechazado la mujer gestante a la solicitud de abortar al feto con el trastorno genético, la mujer dio a luz ambos niños. Los comitentes se llevaron a la niña sana, dejándole a la gestante al niño con alteración genética.

17 The Nation, 2014.

18 U.S. Embassy in Nepal, 2018.

19 The Surrogacy (Regulation) Bill, 2019, p. 19.

${ }^{20}$ Proyecto de ley que regula la gestación por subrogación o gestación subrogada como mecanismo de reproducción asistida, Boletín N 11576-11, Cámara de Diputados, Congreso Nacional, Chile. 
tajante. El órgano legislativo de la Unión Europea condenó esta práctica insistiendo en que "debería prohibirse y analizarse con urgencia en el marco de los instrumentos de derechos humanos" 21 . Además, la Corte Europea de Derechos Humanos resolvió cinco demandas relativas a la negativa de la inscripción del nacimiento de los niños nacidos en el extranjero mediante la gestación por sustitución ${ }^{22}$. En el 2019 la Corte emitió un dictamen consultivo ${ }^{23}$ acerca del reconocimiento de vínculo legal entre el recién nacido y la madre intencional ${ }^{24}$, a raíz del caso Mennesson v. Francia. El Tribunal de Estrasburgo consideró "la necesidad de proporcionar una posibilidad de reconocimiento de la relación legal entre el niño y la madre intencional" 25 , ya que "la imposibilidad de reconocimiento entre el nacido y la madre intencional es incompatible con los intereses más favorables para el niño" 26 . Sin embargo, como indicó el Tribunal, a causa de la falta de consenso acerca de la cuestión entre los Estados miembros del Consejo de Europa, el reconocimiento legal de la relación parental depende de la decisión de cada uno de ellos, atendiendo a los casos en concreto ${ }^{27}$.

Si bien es difícil estimar cuántos niños al año nacen en todo el mundo mediante los contratos de gestación por sustitución, por la falta de accesibilidad a los datos ${ }^{28}$, se considera que la demanda ha aumentado significamente, lo que se refleja en las peticiones de inscripción solicitadas por los comitentes de los niños nacidos en el extranjero de las mujeres gestantes ${ }^{29}$. Lo anterior, debido al incremento de los casos de infertilidad en los países desarrollados, siendo esta una de las causas principales ${ }^{30}$.

Por su parte, también es difícil precisar cuántas parejas del mismo sexo y mujeres u hombres solteros utilizan esta figura para tener un bebé, ya que el acceso a la información en cuanto a la naturaleza, las circunstancias y las estadísticas de este tipo de acuerdos

${ }^{21}$ European Parliament, 2015, pár. 4.

${ }^{22}$ La Corte Europea de Derechos Humanos falló en cinco casos, de estos, cuatro han sido contra Francia y uno contra Italia: TEDH, Mennesson v. Francia de 26 de junio de 2014, n 65192/11; TEDH, Labassee v. Francia de 26 de junio de 2014, n 65941/11; TEDH, Paradiso y Campanelli v. Italia de 27 de enero de 2015, n 25358/12; TEDH, Foulon v. Francia de 21 de julio de 2016, n 9063/14; TEDH, Bouvet v. Francia de 21 de julio de 2016, n 1041/14. En los casos contra Francia (los varones fueron donantes del material genético), la Corte estimó la vulneración de derecho de la vida privada de los recién nacidos, al negar la inscripción de la filiación y el reconocimiento del vínculo legal con el padre biológico, en el sentido del art. 8 del Convenio Europeo de Derechos Humanos. No obstante, aunque la Corte falló a favor de la inscripción de los recién nacidos, los fallos se han basado en el interés superior del menor, en ningún momento refiriéndose a los derechos de las mujeres. En el caso contra Italia, la Corte desestimó la inscripción del niño por no tener este vínculo genético con los comitentes.

${ }^{23}$ Los dictámenes consultivos de la Corte Europea de Derechos Humanos no poseen carácter vinculante.

24 TEDH, Opinión Consultiva del 10 de abril de 2019, solicitud n P16-2018-001.

${ }^{25}$ Op. cit., pár. 47.

${ }^{26}$ Op. cit., pár. 42.

${ }^{27}$ Op. cit., pár. 43.

${ }^{28}$ European Parliament, 2015, p. 32.

29 Véase Freeman, 1990.

30 Peterson, 2014, p. 213. 
difieren dependiendo de la fuente ${ }^{31}$. Según el informe británico respecto de la gestación por sustitución, aproximadamente una quinta parte de los contratos relativos a esta modalidad en Inglaterra se realiza por las parejas del mismo sexo ${ }^{32}$. Aunque no existen cifras oficiales, de acuerdo con la encuesta realizada por Chicago Tribune, se concluyó que entre 10 y $20 \%$ de los óvulos donados por las mujeres son usados para los casos de gestación por sustitución en los que los comitentes son varones homosexuales ${ }^{33}$. Sin embargo, se estima que las parejas heterosexuales son los principales usuarios de esta figura ${ }^{34}$. En este sentido, si bien el perfil de los comitentes puede variar, no cambia el perfil de las gestantes, siendo, en la mayoría de los casos, mujeres pobres de los países en vía de desarrollo ${ }^{35}$. Aunque es cierto que existen mujeres que se ofrecen a gestar un feto de forma voluntaria y altruista, estas situaciones son excepcionales. Un claro ejemplo de lo anterior es el caso del Reino Unido donde se permiten acuerdos de gestación por sustitución de carácter altruista. No obstante, el insuficiente número de mujeres registradas como posibles gestantes frente a la alta demanda, sitúa a los ingleses en la cima de los recurrentes a los contratos comerciales en el extranjero ${ }^{36}$. Lo anterior recalca el carácter excepcional de la gestación por sustitución altruista y deja en evidencia el escaso interés de la práctica en comento.

Así, la gestación por sustitución constituye una figura comercial en la gran mayoría de los países que la permiten. La constante expansión del mercado de subrogación ha generado un vasto aumento de las agencias mediadoras, clínicas de fertilidad o bufetes de abogados especializados en los trámites relacionados con este tipo de contratos. Al final, de la gran parte del lucro que se obtiene a la hora de ejecutar el contrato, se benefician las empresas intermediarias. Siguiendo a Katz, "La gestación por sustitución no ha llegado por vía del progreso tecnológico. Nos ha sido introducida por los bróker -las personas que vieron en ella un nuevo mercado y fueron tras él" ${ }^{37}$-.

De esta forma, el tema analizado está generando una amplia polémica, también dentro del movimiento feminista que está dividido en cuanto a la aceptación de esta figura. En este sentido, su aprobación o rechazo ha estado relacionado con el rol reproductivo de la mujer. Así, el análisis de la gestación por sustitución necesariamente debe abordar elementos claves de esta figura como las consideraciones en torno a la capacidad

31 Véase Horsey, 2015.

${ }^{32}$ Ibid., p. 23. No obstante, no hay datos acerca del número de este tipo de contratos efectuados por las parejas homosexuales en el extranjero.

33 Schoenberg, 2016.

34 Ibid.

35 Véase Crozier, 2010.

36 Véase Valero, 2019, p. 427; Albert, 2017, p. 188. Albert señala que en el Reino Unido, de 271 casos de gestación por sustitución, en 252 casos se recurrió a los países extranjeros, como India o Tailandia. Véase Albert, 2017, p. 189.

A este respecto, Pande señala que los británicos y los estadounidenses constituyen un aplastante número de los compradores en la India. Véase PANDE, 2009.

37 KATZ, 1990, p. 158. 
reproductiva femenina y las tensiones que se verifican entre los planteamientos teóricos feministas de enfoque radical y liberal.

\section{El ROL DE LAS CAPACIDAdES REPRODUCTIVAS DE LA MUJER Y LAS} TENSIONES TEÓRICAS ENTRE EL ENFOQUE FEMINISTA RADICAL Y LIBERAL

La consideración en torno al rol reproductivo de la mujer debe servir como contexto histórico que articula el análisis de la institución. En este sentido, el papel de las habilidades reproductivas femeninas ha sido fuente de la dominación patriarcal durante siglos. Si bien en las últimas décadas, debido a los cambios sociales, políticos y culturales, la procreación biológica es considerada como una de las opciones que tiene la mujer, la figura de la gestación por sustitución refleja el rol con el que la mujer ha estado cumpliendo durante siglos.

Desde esta perspectiva, dar a luz ha sido históricamente considerado como la función primordial de la mujer. Los cambios económicos y sociales que comenzaron en el siglo XVI empezaron a moldear las relaciones jerárquicas y patriarcales entre hombres y mujeres y establecer una división sexual de espacios y trabajo. El apogeo se produjo en la Revolución Francesa, inspirada por la idea del contrato social, cuando, en palabras de Federici, a las mujeres se les excluyó de la vida pública, naturalizando su papel reproductivo ${ }^{38}$. De esta forma, Pateman sostiene que este contrato que creó derechos y libertades para los varones y que se suele presentar como "una historia sobre la libertad"39, estableció también la sujeción de la mujer, comenzando con una sociedad patriarcal ${ }^{40}$. Las mujeres fueron relegadas al espacio privado para cumplir con sus funciones provenientes de la naturaleza donde la maternidad constituía la esencia de la existencia femenina. La autora afirma que el pacto regula no solamente las relaciones que existen entre hombres y mujeres en el sentido patriarcal, sino establece también el derecho que tienen los varones de disponer del cuerpo femenino ${ }^{41}$. En otras palabras, es el contrato sexual que determina los vínculos masculino-femeninos.

Del mismo modo, la capacidad que poseen las mujeres de quedarse embarazadas siempre ha sido controlada por los hombres y ha marcado el rol femenino en la sociedad ${ }^{42}$. Esta dominación respecto de las habilidades reproductivas de las mujeres es un modo de opresión del cual el patriarcado actúa, tratando el cuerpo femenino como propiedad masculina. Como señala De Beavoir, la función reproductiva esclaviza a la mujer, impidiéndole participar en la formación del mundo ${ }^{43}$. En efecto, esta habilidad limita a la mujer a un espacio reducido, previamente determinado mediante un acuerdo de varones.

\footnotetext{
38 FEDERICI, 2006, p. 553.

39 Pateman, 1995, p. 10.

${ }^{40}$ Ibid.

${ }^{41}$ Op. cit., p. 11.

42 Wikler, 1986, pp. 1043, 1050.

43 De Beavouir, 1953, p. 117.
} 
La mujer es definida en términos biológicos en el sentido de sus cargas fisiológicas de reproducción, como "madre en potencia". De esta forma, el patriarcado ha usado las capacidades reproductivas para la construcción social de la mujer, un modelo impuesto como consecuencia de su naturaleza biológica. En esta dimensión, la ideología pronatalista ${ }^{44}$ espera de las mujeres que tengan hijos y en un contexto en donde la sociedad, marcada por las políticas natalistas, les da a entender que la única forma de la que se pueden realizar como mujeres es siendo madres ${ }^{45}$.

Así, la coacción social es parte del control patriarcal de las capacidades reproductivas femeninas que se mantiene hasta hoy y se refleja en la discriminación y rechazo social que sufren las mujeres que deciden abiertamente no tener hijos. El sistema demanda el cumplimiento de las obligaciones biológicas, ejerciendo la presión en las mujeres que intentan desafiar la estructura patriarcal, haciéndolas sentir como "mujeres incompletas". Como bien apunta Saletti, "El deseo de las mujeres no cuenta, porque se supone integrado en el orden de los discursos legitimados del sistema" 46 . Por su parte, ya en los años setenta, Firestone, oponiéndose al sistema, escribió: "En la actualidad, dar abiertamente la cara en contra de la maternidad por principio, es físicamente peligroso para una mujer. Solo puede salir con bien si añade que es neurótica, anormal, odia a los niños, y que, por tanto, "no está en condiciones"' 47.

No obstante lo anterior, en los años setenta del siglo XX, con las nuevas demandas sociales, surgió una nueva política feminista, desafiando el papel reproductivo de la mujer. Los cuestionamientos políticos y culturales del llamado feminismo radical se centraban en las demandas relativas a la libertad sexual, proclamada por Kate Millet y Shulamith Firestone; el derecho al aborto, la anticoncepción, la infertilidad, salud sexual... Así las cosas, el constante progreso tecnológico llevó el debate pertinente a la reproducción iniciado por las feministas radicales, a los temas relacionados con las técnicas de reproducción asistida y gestación por sustitución. En esta dimensión, Firestone sostuvo que la opresión patriarcal que sufren las mujeres tiene su origen en su función reproductiva. Por ello, la única forma de liberarla de esta dominación era crear úteros artificiales y promover tecnologías reproductivas, lo que llevaría a la abolición de la maternidad, la disolución de la figura de familia, logrando la verdadera igualdad entre los sexos ${ }^{48}$. Firestone consideraba la ectogénesis como solución para la desigualdad: un proceso que finalmente aboliría el embarazo, la fuente del dominio masculino que la autora calificaba como "bárbaro". Propuso un sistema alternativo que consistiría en la "liberación de las mujeres de la tiranía de su biología reproductiva por todos los medios

${ }^{44} \mathrm{El}$ concepto de pronatalismo ha tomado diferentes dimensiones en la literatura. Heitlinger sugiere definirlo como "el fomento del nacimiento para favorecer el bienestar individual, familiar y social". Véase Heitlinger, 1993, p. 121.

45 En el mismo sentido, véase Hanmer, 1987, pp. 697-704.

${ }^{46}$ Saletti, 2008, p. 174.

${ }^{47}$ Firestone, 1973, p. 250.

${ }^{48}$ Véase Op. cit., pp. 49, 128, 258. 
disponibles y la ampliación de la función reproductora y educadora a toda la sociedad globalmente considerada, tanto hombres como mujeres" ${ }^{49}$.

Si bien Firestone declaraba el progreso tecnológico en materia de reproducción asistida como un medio de eliminación del patriarcado, en la década de los ochenta se plantearon las teorías acerca del peligro que dichas tecnologías podrían suponer, conduciendo a la explotación de las mujeres. Así, algunas feministas de la segunda ola lo consideraron como un producto del patriarcado y una forma de control ${ }^{50}$. En este sentido, Rowland señala que las profesiones médicas están dominadas por varones, por tanto, el desarrollo de los métodos de reproducción asistida aumenta el control de procreación, significando una mayor dominación social masculina de las mujeres ${ }^{51}$. Corea va más allá y sostiene que la tecnología que está en las manos de los hombres expresa los valores masculinos, como dominación o cosificación, y refuerza el poder masculino sobre las mujeres ${ }^{52}$. No obstante lo anterior, Dworkin mantiene que el problema no es la existencia de las técnicas de reproducción asistida, sino que el uso de ellas dentro de un sistema que trata a las mujeres como objetos que cumplen propósitos meramente sexuales y reproductivos, llevando a que los varones se encuentren en una posición para apoderarse de la totalidad del proceso reproductivo ${ }^{53}$.

Por su parte, Katz apunta que el desarrollo de los métodos de reproducción asistida ha llevado a la práctica del "alquiler de úteros", llamado subrogación, un concepto profundamente arraigado en el patriarcado que rige la reproducción ${ }^{54}$. La autora sostiene que la gestación por sustitución es una forma de explotación femenina y añade que con esta nueva figura algunos privilegios patriarcales se han extendido a las mujeres ricas que con dinero pueden "alquilar un vientre", declarándose propietarias de un bebé gestado por otra mujer ${ }^{55}$. Esta práctica fue criticada y rechazada por las feministas de los años ochenta cuando la gestación por sustitución empezó a generar polémicas, considerándola como una forma de tráfico de niños y comparándola con la prostitución ${ }^{56}$.

Finalmente, el rechazo a las prácticas de reproducción asistida por las feministas radicales se debe al sentimiento de amenaza al cuerpo femenino que llevaría a la explotación de la maternidad, asignando determinados roles a seleccionados grupos de mujeres (gestantes) durante este proceso. De esta forma, el rol que van a cumplir en el proceso de maternidad va a ir determinado por sus aspectos físicos, económicos y sociales.

\footnotetext{
49 Op. cit., p. 258.

${ }^{50}$ DenNy, 1994, pp. 62-63.

51 Rowland, 1987 , p. 524.

52 Corea, 1985 a, p. 4.

53 DwOrKin, 1983, pp. 187-188.

${ }^{54}$ KATZ, 1988, p. 22.

55 Op. cit., p. 23.

${ }^{56}$ En la misma línea, véase RAYMOND, 1989. Andrea Dworkin señala que "La maternidad ha empezado a convertirse en una nueva rama de la prostitución femenina con la ayuda de científicos que desean acceder al útero para la experimentación y el poder (...). Las mujeres pueden vender sus capacidades reproductivas de la misma forma que antiguamente las prostitutas vendían sus capacidades sexuales, pero sin el estigma de prostitución porque no había penetración”. Dworkin, 1983, p. 182.
} 
Corea indica que solo las mujeres con rasgos genéticamente deseables van a poder proporcionar los óvulos, mientras que las mujeres de color o con un nivel socioeconómico bajo van a gestar el feto para que, finalmente, las mujeres ricas, blancas, occidentales puedan tener este bebé y criarlo ${ }^{57}$. Así, las feministas radicales consideraron el uso de las técnicas de reproducción asistida y la gestación por sustitución, una amenaza para la emancipación de las mujeres y una herramienta potente que poseen los varones para seguir consolidando el sistema patriarcal.

No obstante, en oposición a lo declarado por la ola radical, surgió una respuesta proclamada por el feminismo liberal que ha construido desde su óptica los argumentos que se desarrollan en la línea de aceptar la gestación por sustitución. Así, las que abogan por su reglamentación, basando su discurso en la teoría política liberal, la defienden a base de la autonomía individual, la libertad reproductiva y el derecho de decidir libremente, también en cuanto a la celebración de un contrato. En esta línea, Arneson argumenta que esta figura no puede considerarse en los términos del contrato que tradicionalmente establecieron los hombres sobre las mujeres (refiriéndose al contrato sexual planteado por Pateman), ya que el cuerpo de una mujer es suyo y es ella quien decide sí y cómo usarlo ${ }^{58}$. Por su parte, Andrews considera que la gestación por sustitución constituye una de las formas de reproducción que una mujer debería poder elegir libremente, manifestando que "El desarrollo reproductivo se ha sumado a la libertad de ser gestante" 59 . El hecho de optar por gestar un feto para otras personas constituye una forma de evolución natural del derecho de elegir de forma libre sobre la reproducción ${ }^{60}$. Por su parte, Gostin argumenta que una mujer sabe lo que es mejor para ella y en el caso de la gestación por sustitución, toma una decisión autónoma que está en su mejor interés, por tanto, cualquier impedimento legal que el Estado podría realizar, sería una intervención de carácter paternalista ${ }^{61}$. En la misma línea, Arneson, apoyando el argumento de la autonomía de la mujer gestante y la libertad de elegir, sostiene que "Sin importar cuánto sean restringidas las opciones de la vida de una persona, la idea de que el reducido número de alternativas que esta persona tiene, limita inaceptablemente su elección y no establece la razón de limitar aún más su rango de elección" ${ }^{62}$. Por tanto, cualquier limitación vulneraría su derecho a la autonomía ${ }^{63}$, lo que, en palabras de Hatzis, sería dañino para las mujeres ${ }^{64}$.

Esta perspectiva sitúa a la gestación por sustitución en la misma línea que cualquier otra forma de trabajo retributivo. Hatzis afirma que una mujer gestante vende sus

${ }^{57}$ Corea, 1985b, pp. 299-305. En el mismo sentido, véase también Dworkin, 1983.

58 Arneson, 1992, p. 162.

59 Andrews, 1988, pp. 167-168. En la misma línea, Gostin la considera como una opción más que ofrece la tecnología. Gostin, 1988.

${ }^{60}$ Lieber, 1992, p. 227.

${ }^{61}$ Gostin, 1988, p. 15.

62 Arneson, 1992, p. 158.

63 SIFRIS, 2015, p. 369.

${ }^{64}$ Hatzis, 2003, p. 423. 
servicios gestacionales, comparándolo con el trabajo que realiza una actriz o una niñera, indicando que en este caso solamente usa su útero ${ }^{65}$. Por su parte, Gostin defiende la idea de gestar un feto para otros, llamándolo un "trabajo femenino" 66 que tiene "un alto valor social" y lo considera "un trabajo valioso" 67 . El autor hace hincapié en que el pago por un servicio efectuado es una cuestión esencial en nuestra sociedad ${ }^{68}$. En la misma línea, Sifris entiende a la gestación por sustitución como un servicio reproductivo que se realiza a cambio de un monto de dinero y que constituye una manifestación de su derecho a trabajar, por tanto, no se debe cuestionar ${ }^{69}$.

Así, los que apoyan a la gestación por sustitución, la ven como una forma de trabajo que se establece mediante un contrato. Hatzis considera que el cumplimiento del dicho contrato resulta ventajoso para ambas partes, ya que los comitentes obtienen al bebé y la mujer gestante el dinero con que el que puede pagar la educación de sus hijos ${ }^{70}$. Por tanto, este tipo de contrato puede constituir una oportunidad económica para la mujer ${ }^{71}$. Esta línea argumentativa presentada por el feminismo liberal hace hincapié en la capacidad económica que tiene la mujer para cuidar de su familia aportando dinero, mediante el disfrute de la libertad de usar su cuerpo, la libertad de elección y la libertad contractual ${ }^{72}$. Por tanto, el argumento de que las mujeres que deciden ser gestantes tienden a ser mujeres de bajos recursos que carecen de otras opciones laborales, no constituye una razón válida, por la que este tipo de contratos debería prohibirse ${ }^{73}$. En la relación gestante-comitente no se puede hablar de explotación, porque existe una compensación por el servicio efectuado ${ }^{74}$.

En definitiva, el fundamento del enfoque liberal consiste en la percepción de que gestar un feto para otros se basa en un contrato de dos partes libres que se benefician de ello mutuamente, viendo en la gestación por sustitución una figura que representa autonomía, libertad reproductiva y el goce del derecho a la libertad de tomar decisiones sobre el uso del cuerpo. Esta dimensión se centra en el enfoque contractual, civil, patrimonial y económico, desconociendo lo que en realidad se debería analizar considerando esta figura, que son los derechos de la mujer. Los que abogan por la legalización de la gestación por sustitución, tienden a desconocer otros aspectos relevantes que dicen relación con su desarrollo, tales como la perspectiva histórica relativa a la posición desigual de la mujer que se ha mantenido a lo largo de los siglos, obviando el hecho de que se está usando el útero de un sujeto que históricamente no ha estado empoderado. Así, en el

\footnotetext{
${ }^{65}$ Op. cit., pp. 420-421.

${ }^{66}$ En la misma línea, Andrews, 1989.

${ }^{67}$ Gostin, 1988, p. 15. En la misma línea, LEWIS,

68 Op. cit., p. 11.

${ }^{69}$ SIFRIS, 2015, p. 369.

${ }^{70}$ Hatzis, 2003, p. 417.

71 Véase Huber, 2017.

72 Sills, 2016, p. 26.

73 Arneson, 1992, p. 158.

${ }^{74}$ Sifris, 2015, p. 368. En la misma línea, Gostin, 1988, p. 10.
} 
contexto actual donde el feminismo tiende a fomentar el papel de la mujer en todos los ámbitos, los argumentos que ofrece el feminismo liberal no contribuyen a esta finalidad.

El planteamiento liberal tampoco toma en consideración el contexto socioeconómico marcado por una severa desigualdad en el que se encuentra la mayoría de las mujeres gestantes. En consecuencia, desconoce u omite en su discurso un punto clave para el análisis de esta figura que es la operatividad y el funcionamiento de esta, sin tener en cuenta que en los países que regulan de forma permisiva la gestación por sustitución, finalmente, el agente de la institución no es la gestante sino las empresas intermediarias que captan a las más vulnerables, quedándose con un alto margen de ganancias de la ejecución del contrato ${ }^{75}$.

Lo anterior, al contrario de la postura que representan las feministas radicales y que sobrepone los derechos de las mujeres por sobre cualquier otro aspecto relacionado con esta figura. Sus estudios consideran a la gestación por sustitución como una forma de opresión patriarcal de la mujer, analizándola desde una perspectiva histórica del sometimiento de las capacidades reproductivas femeninas al poder de los varones. Apuntan en que las técnicas de reproducción asistida consolidan el dominio patriarcal de los hombres sobre las mujeres y las convierten en un mero objeto que se puede alquilar para gestar un feto. En este sentido, nos encontramos ante el enfoque propio de derecho donde no se discute los derechos patrimoniales sino fundamentales.

\section{LA GESTACIÓN POR SUSTITUCIÓN: UN DEBATE CRÍTICO SOBRE EL NUEVO NEGOCIO GLOBAL}

La posición de las feministas liberales acerca de la gestación por sustitución es objetable y tiene que ser relativizada no únicamente por los argumentos que surgieron dentro del feminismo radical después de la revolución sexual. Para ello, es imprescindible tomar también en consideración todos aquellos que se plantean por las feministas actuales y que contribuyen a objetar la posición que aboga por la regularización de esta institución. En este sentido, hay que poner en evidencia que este tipo de contrato se caracteriza por una desigualdad social donde las personas ricas de Occidente viajan a los países de un bajo nivel de desarrollo socioeconómico en los que la oferta de mujeres gestantes es mucho más vasta y el costo del servicio incomparablemente menor que en los países occidentales que regulan esta práctica ${ }^{76}$. El poder económico permite además

75 Véase Gruber, 2013. Varias clínicas en India buscan a las mujeres dispuestas a gestar un feto para otros entre los grupos más vulnerables. Por su parte, se estima que, en Estados Unidos, alrededor de $50 \%$ de las gestantes son esposas de los militares que tienden ser personas de bajos recursos, y, por tanto, son el principal objetivo de las agencias intermediarias.

76 Estados Unidos es el destino más caro para los comitentes donde el precio se establece entre los 100.000-150.000€. En Ucrania, uno de los destinos más populares para los europeos, el precio oscila entre 35.000-55.000€. Por su lado, en algunos países asiáticos, como Camboya, el precio establecido está entre 20.000-30.000€. Véase Instituto Vasco de la Mujer, 2018, p. 53. 
elegir el sexo del que está por nacer, y en el caso de que sea necesaria la donación de óvulos, el precio va a variar dependiendo de si la donante es caucásica o india ${ }^{77}$. Esta nueva variación de libre mercado que oculta el verdadero problema de explotación, pobreza y desigualdad permite que las personas ricas puedan negociar condiciones del contrato en función de su capacidad económica. En este sentido, Satz califica el mercado de subrogación como un mercado nocivo determinado por una posición desigual entre las partes y que se basa "en la desesperación, la humillación o la súplica, o cuyos términos de redención involucran la servidumbre o la esclavitud ${ }^{78 ”}$. La autora explica que este tipo de mercados proceden de la pobreza y las personas que los integran "tienen una necesidad muy desigual de los bienes que son objeto de intercambio"79.

Por su parte, varios factores influyen en la situación socioeconómica de las mujeres y son el impulso para tomar la decisión de ser gestante. La posición de desventaja en la que se encuentran en el mercado laboral está históricamente marcada por su rol social que les fue adjudicado siglos atrás y que pertenece al ámbito privado, y que, a pesar de la progresiva conquista de derechos, sigue latente en la sociedad. Las labores domésticas, el cuidado de hijos y ancianos, falta de formación como consecuencia de lo anterior, o tradiciones culturales y familiares, en la mayoría de los casos impiden a que la mujer consiga una estabilidad laboral o incluso, en varios casos, le imposibilita el ingreso al mercado de trabajo. A este respecto, una persona que se encuentra en una situación vulnerable que genera circunstancias de necesidad, va a aceptar una oferta cuyas condiciones no serían favorables si no se hallara en esta posición ${ }^{80}$. Es muy poco probable que una mujer con formación y estabilidad económica se decida a gestar un feto para otros. Por ello, la práctica de gestación por sustitución lo que hace es profundizar el ya extenso problema de la feminización de la pobreza ${ }^{81}$, obligando a las mujeres llegar al límite y poner a disposición del mercado lo último que les queda para poder subsistir que es su cuerpo. Así, la situación social, política y económica generada por el Estado impone a las mujeres vender sus capacidades reproductivas para poder sobrevivir ${ }^{82}$, obligando a tomar decisiones que en el mundo occidental se disfrazan de "opciones" 83 . En cualquier caso, en palabras de Ekman, la industria de la gestación por sustitución "alimenta la autodestrucción femenina" 84 .

\footnotetext{
77 Op. cit., p. 4.

78 SATZ, 2015, pos. 108.

79 Op. cit., pos. 114.

${ }^{80}$ Op. cit., pos. 112.
}

${ }^{81}$ Josepf E. Stiglitz indica que los salarios de las mujeres son significamente más bajos que los de los hombres. Así, señala que en el 2010 el salario de una mujer correspondía al $80 \%$ del salario de un hombre. Véase Stiglitz, 2012, pp. 139, 491.

82 Corea, 1985a, p. 228.

${ }^{83}$ En el mismo sentido, en India o Nepal, la gente vende sus riñones en el mercado negro para poder subsistir. Véase Actualidad, 2015.

${ }^{84}$ EKMAN, 2017, p. 226. 
Así, de acuerdo con lo señalado anteriormente, la relación comercial que existe entre las partes del contrato de subrogación se desarrolla en un contexto en el que predomina la asimetría económica, social y educacional entre ambas: los comitentes suelen ser personas más adineradas y mejor educadas que las gestantes ${ }^{85}$, y estas últimas normalmente provienen de los países en vía de desarrollo que viven en la extrema pobreza. Dicha relación desproporcionada crea lo que Guerra llama la "explotación reproductiva" ${ }^{86}$. En este sentido, el mercado de subrogación produce un efecto peligroso que lleva a la explotación, tráfico de mujeres y secuestros de niños ${ }^{87}$. En el caso de varios países asiáticos, la estructura patriarcal de la sociedad somete a las mujeres a la explotación laboral. En el contexto global en el que se encuentran estas mujeres, la opción de decidir libremente se convierte en una obligación. Por ello, analizar el tema de la gestación por sustitución en abstracto corre peligro de dejar al margen los aspectos más relevantes de esta discusión.

Desde esta perspectiva, el mercado patriarcal ha creado un nuevo tipo de contrato que sigue los antiguos patrones de sujeción de la mujer. Como ya he señalado bien, las mujeres siempre han sido consideradas objetos sexuales para la explotación masculina. Así, el contrato sexual que firmaron los varones hace casi 400 años, hoy toma una nueva forma. Pateman entiende la gestación por sustitución como otro modo del contrato sexual donde el varón tiene el pleno acceso al cuerpo femenino y lo explota. El cliente es el hombre que aporta su semen para que la mujer gestante pueda dar a luz a cambio de una retribución económica. Lo anterior constituye una nueva forma de patriarcado donde, por medio de un contrato, se produce un abuso del cuerpo femenino por parte del poder masculino ${ }^{88}$. Como apunta Satz, "Este tipo de contratos convierte el trabajo de la mujer en algo que puede ser empleado y controlado por otros, y así valida estereotipos de género utilizados a lo largo de la historia para justificar el estatus desigual de la mujer" La explotación está disfrazada de "libre elección", apoyándose en la liberación femenina para poder plantear el tema de la gestación por sustitución, lo que nunca se plantearía con, por ejemplo, la compraventa de órganos humanos. En este sentido, Nuño señala:

"El libre consentimiento es el mejor mantra que se utiliza cada vez que hay que explotar a las mujeres. El libre consentimiento no se utiliza cuando se habla de esclavitud, ni de trabajar 40 horas al sol y con cadenas, ni de la venta de órganos, ni de las relaciones laborales... Sin embargo, es un mantra que sí se utiliza curiosamente cuando se habla de prostitución, de vientres de alquiler... es un mantra

${ }^{85}$ European Center For Law And Justice, 2012, p. 3. En el mismo sentido, véase Centre For Social Research, 2010.

86 Guerra, 2017, p. 538.

87 Véase SARAVANAN, 2018.

88 Pateman, 1995, pp. 297, 299.

89 SATZ, 2015, pos. 147. 
que solo se utiliza cuando los derechos en juego son los de las mujeres, pero no para otro tipo de derechos" 90 .

De Miguel lo asocia con la nueva forma económica e ideológica del poder patriarcal que es el neoliberalismo sexual, explicando que el límite del mercado lo va a marcar el libre consentimiento individual. No obstante, estas decisiones libres se toman en el contexto de una desigualdad estructural que atraviesa a la sociedad. El mito de la libre elección del que habla De Miguel constituye una trampa del sistema patriarcal que convierte el cuerpo femenino en mercancía ${ }^{91}$. En el mismo sentido, Balaguer advierte que en el contexto de emancipación y autodeterminación femenina que crea la actual legislación, se esconde "un nuevo tipo de esclavitud sexual"92.

En esta dimensión, el contrato desigual que suscribe la gestante con los comitentes limita la libertad de esta primera, ya que le suele imponer ciertas condiciones con las que la mujer debe cumplir durante el proceso. Así, en varios casos, tiene obligación de abstenerse de fumar y tomar alcohol durante el embarazo, someterse periódicamente a los exámenes para establecer si el feto tiene algunas deficiencias (en el caso de que exista alguna anomalía, existen cláusulas que contemplan aborto a petición de los comitentes), abstenerse de mantener relaciones sexuales en determinadas etapas del embarazo, finalmente dar a luz por cesárea ${ }^{93}$, entre otros. A esta razón, hay que añadir la falta del consentimiento informado acerca del proceso de gestación y las posibles consecuencias, ya que el bajo nivel educativo de las mujeres en los países en vía de desarrollo, en varias ocasiones les impide entender el contrato que firman. En la mayoría de los casos, las mujeres no saben leer ni escribir. A este respecto, en las sociedades patriarcales son los varones (padres, maridos, familias enteras) quienes toman todas las decisiones, dejando de lado la voluntad de las mujeres. Por ello, en el caso de gestación por sustitución, son ellos quienes autorizan el proceso. En consecuencia, la decisión libre de la mujer se pone en duda ${ }^{94}$. Lara señala que el utilizado argumento de libre consentimiento de la mujer para gestar un feto para otras personas está viciado y no se puede considerar válido, ya que su decisión no es realmente libre, teniendo en cuenta que la mujer se encuentra en unas condiciones extremas ${ }^{95}$. Como bien señala Satz, el contrato de subrogación legitima el sometimiento del cuerpo femenino al control de otros, lo que hace que la desigualdad de género se siga manteniendo 96 . En este sentido, no podemos hablar de libertad cuando alguien toma una decisión impulsado por las circunstancias en las que se encuentra, y como última opción que tiene para poder sobrevivir. Lo anterior se presencia aún más en

${ }^{90}$ Laura Nuño Gómez, entrevista realizada en el marco del estudio del Instituto Vasco de la Mujer, 2018, p. 49.

91 Véase De Miguel, 2015.

92 Balaguer, 2017, p. 178.

93 Véase Fernández 1988, pp. 647-683, 657 y Bellver, 2015, p. 45.

${ }^{94}$ Instituto Vasco de La Mujer, 2018, p. 65.

95 LARA, 2018.

96 SATZ, 2015 , pos. 153. 
el caso de que la gestante al final optara por quedarse con el bebé, ya que determinadas legislaciones, como la israelí, no lo prevé. Además, en el caso de las legislaciones que aceptan esta opción, el nivel socioeconómico de la gestante probablemente le impediría hacerse cargo del recién nacido. Además, el invocado (por los que defienden la legitimidad de la gestación por sustitución) derecho a decidir de su propio cuerpo, se convierte en una utopía, ya que, con las cláusulas que contiene el contrato, al final quien decide acerca del cuerpo de la gestante, son los comitentes ${ }^{97}$.

A todo ello, es curioso cómo cambia el panorama de discusión cuando lo que está en el juego es el lucro. Desde hace años, las demandas feministas en todo el mundo están invocando el derecho de decidir de su propio cuerpo en la lucha por la legalización del aborto. Este les ha sido negado constantemente, basándose en el argumento de que la mujer comparte el cuerpo con un nuevo ser y que no se puede invocar la libertad del cuerpo para decidir por la vida de otro. Por tanto, se trata de un discurso selectivo y contradictorio, ya que, en ocasiones, el ejercicio de la autonomía para decidir respecto del cuerpo se predica en favor de la legalización de la gestación por sustitución, sin embargo, cuando se relaciona con el aborto, se invoca la negación del mismo. Dentro de ese marco, el hecho de alegar el derecho a la libre elección para justificar la legitimidad de la gestación por sustitución, en realidad encubre la mercantilización del cuerpo femenino y la explotación reproductiva, siguiendo los antiguos patrones patriarcales de objetivación de la mujer que hoy persisten en las voces que insisten de que las mujeres son libres y autónomas.

En consecuencia, habiendo hecho una discusión teórica desde la figura, se invita a considerar estos planteamientos para una eventual regulación legislativa de la misma. En efecto, a partir de los elementos abordados, la presente investigación deja abierta una dimensión de análisis que tenga por objeto pensar en el diseño jurídico que podría configurarse para regular la eventual aceptación o prohibición de la gestación por sustitución $^{98}$.

97 VALERo, 2019, pp. 421-440, 432.

${ }^{98}$ Los países que expresamente prohíben los contratos de gestación por sustitución establecen, en su mayoría, una norma prohibitiva civil con la consecuencia de la invalidez del contrato, tal y como se dispone, por ejemplo, en el caso de España, en la Ley 14/2006 LTRA; en el caso de Francia, en los artículos 16-17 del Código Civil, introducidos mediante la Ley n 94/653; en el caso de Italia, en la Ley $n^{\circ} 40$ de 2004 , sobre normas en materia de procreación médica asistida. Véase Lamm, pp. 71, 118, 127. En los países que permiten la gestación por sustitución de carácter altruista, la legislación prevé una retribución por los gastos derivados del embarazo y el parto. Este es el caso de, por ejemplo, Canadá o Reino Unido. La sección 6 de la ley canadiense Assisted Human Reproduction Act 2004 (c. 2) expresamente prohíbe la gestación por sustitución comercial, condenando las partes involucradas en un acuerdo de este tipo a sanciones penales de hasta \$500.000 de multa o 10 años de privación de libertad. En el caso del Reino Unido, la ley The Surrogacy Arrangements Act 1985 (c. 49) en su artículo 2 (1) y (2) considera delito facilitar o negociar un acuerdo relativo a la gestación por sustitución comercial y publicitar la posibilidad de estos acuerdos. 


\section{COnClusiones}

El creciente mercado de la gestación por sustitución ha generado una compleja discusión dentro del movimiento feminista acerca de la aceptación o rechazo de esta figura. El argumento que domina en la ideología feminista liberal relativo a la libertad de tomar decisiones por el uso del cuerpo y de celebrar contratos, ha sido firmemente refutado por el grupo de feministas que consideran esta figura como una forma de explotación de la mujer más vulnerable. Una persona no puede ejercer autónomamente sus derechos a la libertad de elección si se encuentra en una posición desigual. Por ello, las decisiones de gestar un feto para otros que toman las mujeres que se encuentran en una situación de vulnerabilidad, no se pueden considerar libres.

La situación de necesidad de ambas partes ha sido utilizada por el mercado que ha visto una oportunidad de ganancia en la figura de gestación por sustitución. El hecho de prevalecer en nuestra sociedad valores conservadores y sumando al prototipo de la mujer como madre, muchas veces lleva a las personas a cumplir con las expectativas sociales a toda costa. Por su parte, el sistema capitalista ha creado una nueva forma de negocio, aprovechándose de la desesperación de, por un lado, las personas infértiles que por la presión social y familiar hacen todo lo posible para poder tener un hijo con el mismo ADN que ellos, y, por otro lado, de las mujeres pobres que, en gestar a un feto para otros, encuentran la única forma de sobrevivir. En este sentido, el sistema convierte a las personas en mercancías, generando cada vez mayor desigualdad social entre los países occidentales y los que están en vía de desarrollo.

En efecto, como se ha demostrado a lo largo de la historia, las víctimas del sistema han sido siempre las mujeres. Así, existe un común asombro y rechazo por parte de la sociedad a la situación de las mujeres "criadas" del distópico libro de Margaret Atwood titulado El cuento de la criada ${ }^{99}$, donde ellas están obligadas a dar a luz para las parejas de "los comandantes" - los más altos jerárquicos de la sociedad de Gilead-. Sin embargo, no incomoda el hecho de que las personas que se encuentren en una posición económica ventajosa se aprovechen de la situación de vulnerabilidad de las mujeres en situación de necesidad para satisfacer sus deseos, recurriendo a la gestación por sustitución. Finalmente, se tiende a generalizar la idea de que los temas relativos a la gestación por sustitución se circunscriben a una relación patrimonial de prestación de un servicio por el que se paga un precio determinado, subordinando su contenido a reglas ius privatistas y no desde la dimensión de los derechos de las mujeres. Desde esta perspectiva, la legislación permisiva de la gestación por sustitución constituye un peligro de desarrollo de una práctica de mercantilización del cuerpo. Además, supone un aumento de la brecha socioeconómica donde la gente se va a sentir en todo el derecho de comprar cualquier cosa, hasta la vida de un recién nacido, y las personas pobres van a ofrecer lo último que tienen que es su cuerpo, para poder sobrevivir. 
A mi juicio, la legislación prohibitiva de la gestación por sustitución puede influir en el resguardo de los derechos de las mujeres más vulnerables, estableciendo un límite a un contrato, aunque sea altruista, siendo este, el mismo cuerpo de la mujer. Si esta práctica se normaliza al amparo de la ley, se corre el peligro de fomentar una maquinaria de la industria reproductiva que será difícil de frenar, situándonos un paso más cerca del mundo creado por Aldous Huxley en la obra Un mundo feliz ${ }^{100}$ que hace casi 90 años parecía una utopía pero que hoy cada vez es más real.

\section{BIBLIOGRAFÍA}

ACTUALIDAD, 2015: "La aldea nepalesa en la que casi todos vendieron un riñón para comprar una casa".

Disponible en https://actualidad.rt.com/sociedad/179809-aldea-nepal-vender-rinon-comprar-casa

Albert Márquez, Marta, 2017: "La explotación reproductiva de mujeres y el mito de la subrogación altruista: Una mirada global al fenómeno de la gestación por sustitución”, Cuadernos de Bioética, Vol. 28, $\mathrm{N}^{\circ} 93$.

Andrews, Lori, 1988: "Surrogate Motherhood: The Challenge for Feminists", The Journal of Law, Medicine E Ethics, Vol. 6, $\mathrm{N}^{\circ} 1-2$.

Andrews, Lori, 1989: Between Strangers: Surrogate Mothers, Expectant Fathers, and Brave New Babies, Nueva York: Harper \& Row.

Arneson, Richard, 1992: "Commodification and Commercial Surrogacy", Philosophy \& Public Affairs, Vol. 21, $N^{\circ} 2$.

AssisTED Human Reproduction Act, SC 2004, c 2, Canadá.

ATwood, Margaret, 2017: El cuento de la criada, Barcelona: Narrativa Salamandra.

Ávila Hernández, Carlos Javier, 2017: "La maternidad subrogada en el Derecho comparado”, Cadernos de Dereito Actual, No6.

Balaguer, María Luisa, 2017: Hij@s del mercado. La maternidad subrogada en un Estado Social, Valencia: Ediciones Cátedra.

Bellver Capella, Vicente, 2015: “¿Nuevas tecnologías? Viejas explotaciones. El caso de la maternidad subrogada internacional”, SCIO. Revista de Filosofía, $\mathrm{N}^{\circ} 11$.

CEntre for Social Research, 2010: "Surrogate Motherhood: Ethical or Commercial”. Disponible en https://archive.nyu.edu/bitstream/2451/34218/2/Surrogacy-Motherhood-Ethical-orCommercial-Surat\%2cGujraat.pdf

Corea, Gena, 1985a: The Mother Machine, London: The Women’s Press.

Corea, Gena, 1985b: "How the New Reproductive Technologies Could Be Used to Apply the Brothel Model of Social Control Over Women", Women's Studies International Forum, Vol. $8, \mathrm{~N}^{\circ} 4$.

Cottingham, Jane, 2017: "Babies, Border and Big Business”, Health Matters, Vol. 25, No49.

Crozier, Gilian, 2010: "Protecting cross-border providers of ova and surrogacy services?", Global Social Policy, Vol. 10.

DE Beavouir, Simone, 1953: The second sex, New York: Knopf.

De Miguel Álvarez, Ana, 2015: El neoliberalismo sexual. El mito de la libre elección, Madrid: Ediciones Cátedra. 
DenNy, Elain, 1994: "Liberation or opression? Radical feminism and in vitro fertilisation", Sociology of Health E Illness, Vol. 16, $\mathrm{N}^{\circ} 1$.

Dworkin, Andrea, 1983: Right-Wing women, New York: Perigee.

EKMAN, Kajsa Ekis (25 de febrero de 2016). All surrogacy is exploitation. The world should follow the Sweden's ban. Recuperado de The Guardian: https://www.theguardian.com/commentisfree/2016/ feb/25/surrogacy-sweden-ban

Ekman, Kajsa Ekis, 2017: El ser y la mercancía. Prostitución, vientres de alquiler y disociación, Barcelona: Bellaterra.

European Center For Law And Justice, 2012: "Surrogate motherhood: a violation of human rights", European Center for Law and Justice, Report presented at the Council of Europe, Strasbourg, on 26 April 2012.

European Parliament, 2013: A comparative study on the regime of surrogacy in EU Member States. Recuperado de https://www.europarl.europa.eu/RegData/etudes/STUD/2013/474403/ IPOL-JURI_ET(2013)474403_EN.pdf

European Parliament, 2015: Report on the Annual Report on Human Rights and Democracy in the World 2014 and the European Union's policy on the matter, 2015/2229(INI). FIRESTONE, Shulamith, 1973: La dialéctica del sexo. En defensa de la revolución feminista, Barcelona: Editorial Kairos.

Federici, Silvia, 2006: “Women, Gender Oppression, and Science”, Science E Society, Vol. 70, ํ 4. Fernández Pacheco, María Teresa, 1988: "La maternidad subrogada en Norteamérica: la Sentencia de Baby M", RGLJ, Vol. 5.

Freeman, Michael D.A., 1990: Is surrogacy exploitative?, en McLean, Sheila (ed) Legal Issues in Human Reproduction, Hanover: Dartmouth Publishing.

Gostin, Lawrence, 1988: “A Civil Liberties Analysis of Surrogacy Arrangements”, L. Med. E Health Care, Vol. 16.

Gruber, Jeremy, Krimsky, Sheldon, 2013: Biotechnology in Our Lives: What Cutting-Edge Genetic Research Can Tell You about Gene Patents, Human Cloning, Assisted Reproduction, Predicting Criminal Behavior, Bioweapons, and Much More, Nueva York: Skyhorse Publishing.

Guerra Palmero, María José, 2017: “Contra la llamada gestación subrogada. Derechos humanos y justicia global versus bioética neoliberal”, Gaceta Sanitaria, Vol. 31, Nº 6 .

Hanmer, Jalna, 1987: "Reproduction trends and the production of moral panic", Social Science and Medicine, Vol. 25, $\mathrm{N}^{\circ} 6$.

Hatzis, Aristides, 2003: “Just the Oven': A Law \& Economics Approach to Gestational Surrogacy Contracts", en Boele-Woelki, Katharina (ed), Perspectives for the unification or harmonisation Of Family Law in Europe, Antwerp: Intersentia.

Heitlinger, Alena, 1993: Women's Equality, Demography and Public Policies A Comparative Perspective, Houndmills: Macmillan Press.

Horsey, Kirsty, 2015: "Surrogacy in the UK: Myth busting and reform Report of the Surrogacy UK Working Group on Surrogacy Law Reform”. Disponible en https://kar.kent.ac.uk/59740/

Huber, Sarah, Karandikar, Sharvari, Gezinski, 2017: "Exploring Indian Surrogates' Perceptions of the Ban on International Surrogacy", Affilia: Journal of Women and Social Work, Vol. 33, $\mathrm{N}^{\circ} 1$.

Huxley, Aldous, 2013: Un mundo feliz, Madrid: Cátedra.

Instituto Vasco de la Mujer, España, 2018: “¿Gestación subrogada o vientres de alquiler? Informe final”. Disponible en http://emakunde.blog.euskadi.eus/wp content/uploads/2018/07/ ANEXOENTREVISTAS21042018.pdf

Katz Rothman, Barbara, 1988: “Cheap labor: Sex, Class, Race- and 'Surrogacy”, Society, Vol. $25, \mathrm{~N}^{\circ} 3$.

KaTz Rothman, Barbara, 1990: Recreating Moterhood: Ideology and Technology in a Patriarcal Society, Nueva York: Norton. 
LAMm, Eleonora, 2013: Gestación por sustitución. Ni maternidad subrogada ni alquiler de vientres, Barcelona: Universitat de Barcelona.

LAMM, Eleonora, 2017: “Argumentos para la necesaria regulación de la gestación por sustitución”, Gaceta Sanitaria, Vol. 31, N 6.

LARA Aguado, Ángeles, 2018: "La gestación subrogada ¿Una forma de liberación o de esclavitud de la mujer?", Revista Iberoamericana de Derecho Internacional y de la Integración, $\mathrm{N}^{\circ} 8$. Disponible en http://ar.ijeditores.com/articulos.php?Hash=7baf8dac7295ae122974b9722 1ea981b\&hash_t $=11019 a 87283 c c 2 b b 2 d a 2364 e 3 c 3 b 7 d 29$

Lehman, Jeffrey, Phelps, Shirelle, 2005: West's encyclopedia of American Law, Detroit: Gale.

LEWIS, Sophie, 2019: Full Surrogacy Now: Feminism Against Family, Nueva York: Verso Book.

LEY 14/2006, Sobre técnicas de reproducción humana asistida, publicada con fecha 28 de mayo de 2006, BOE, España.

LEY N $\mathrm{N}^{\circ}$ 94-653, Sobre el respeto del cuerpo humano, publicada con fecha 29 de julio de 1994 , Francia. Ley $n^{\circ} 40$, Sobre normas en materia de procreación médica asistida, publicada con fecha 19 de febrero de 2004, Italia.

Ministerio de Salud, Chile, s/f. Disponible en https://yodonovida.minsal.cl/estadisticas/numerotrasplantes.html

Ministri: Health and Family Welfare (India), 2019: The surrogacy (regulation) Bill. Disponible en http://prsindia.org/billtrack/surrogacy-regulation-bill-2019

Mohapatra, Seema, 2012: "Stateless Babies \& Adoption Scam: A Bioethical Analysis of International Commercial Surrogacy”, Berkeley J. Int'l L. Vol. 30.

Organización Mundial de la Salud, 1991: Principios Rectores de la OMS sobre trasplante de células, tejidos y órganos humanos. Resolución WHA44.25, de la Asamblea Mundial de la Salud.

Pande, Amrita, 2009: "Not an 'Angel', not a 'Whore': Surrogates as 'Dirty' Workers in India”, Indian Journal of Gender Studies, Vol. 16, $\mathrm{N}^{\circ} 2$.

Pateman, Carole, 1995: El contrato sexual, Barcelona: Anthropos.

Peterson, Kate, 2013: Cross Border Commercial Surrogacy, en: Freeman, Michael, Hawkes, Sarah, Bennett, Belinda (ed) Law and Global Health: Current Legal Issues, Vol. 16.

Plataforma No somos vasijas. Recuperado de http://nosomosvasijas.eu/

Proyecto de ley que regula la gestación por subrogación o gestación subrogada como mecanismo de reproducción asistida, Boletín 11576-11.

RAYMOND, Janice, 1989: "The international traffic in women: Women used in systems of surrogacy and reproduction”, Reproductive and Genetic Engineering, Vol. 2, N 3 .

Rowland, Robyn, 1987: "Technology and motherhood: reproductive choice reconsidered", Signs, Vol. 12, $\mathrm{N}^{\circ} 3$.

SALETTI Cuesta, Lorena, 2008: "Propuestas teóricas feministas en relación al concepto de maternidad", Clepsydra, Vol. 7.

SANNHEz Aristi, Rafael, 2010: "La gestación por sustitución: dilemas éticos y jurídicos", Humanitas, Humanidades médicas, $\mathrm{N}^{\circ} 49$.

Saravanan, Sheela, 2018: A Transnational Feminist View of Surrogacy Biomarkets in India, Singapour: Springer.

SAtz, Debra, 2015: Por qué algunas cosas no deberían estar en venta. Los límites morales del mercado, Buenos Aires: Siglo veintiuno.

Schoenberg, Nara, 2016: "Gay Men Increasingly Turn to Surrogates to Have Babies", Chicago Tribune. Disponible en www. chicagotribune.com/lifestyles/health/sc-gay-men-havingbabieshealth-1130-20161123-story.html

Sifris, Ronli, 2015: "Commerial Surrogacy and the Human Right to Autonomy", J Law Med, Vol. 23, $\mathrm{N}^{\circ} 2$. 
Sills, Scott, 2016: Handbook of gestional surrogacy, Cambridge: Cambridge University Press.

Sreenivas, Kiran, Campo-Engelstein, Lisa, 2010: "Domestic and International Surrogacy Laws: Implications for Cancer Survivors", Cancer Treat Res. N 156.

STiglitz, Joseph E., 2012: El precio de la desigualdad, Madrid: Taurus.

Tribunal Europeo de Derechos Humanos, sentencia Mennesson v. Francia de 26 de junio de 2014, N 65192/11.

Tribunal Europeo de Derechos Humanos, sentencia Labassee v. Francia de 26 de junio de 2014, $\mathrm{N}^{\circ} 65941 / 11$.

Tribunal Europeo de Derechos Humanos, sentencia Paradiso y Campanelli v. Italia de 27 de enero de 2015, $\mathrm{N}^{\circ} 25358 / 12$.

Tribunal Europeo de Derechos Humanos, sentencia Foulon v. Francia de 21 de julio de 2016, $\mathrm{N}^{\circ} 9063 / 14$.

Tribunal Europeo de Derechos Humanos, sentencia Bouvet v. Francia de 21 de julio de 2016, $\mathrm{N}^{\circ} 1041 / 14$.

Tribunal Europeo de Derechos Humanos, Opinión Consultiva del 10 de abril de 2019, solicitud $\mathrm{N}^{\circ} \mathrm{P} 16-2018-001$.

The Nation, 2014: "Commercial Surrogacy Bill Passes First Reading with 177 to 2 Votes". Disponible en https://perma.cc/3SYB-4LYH

The Surrogacy Arrangements Act 1985 (c. 49), Reino Unido.

U.S. Embassy In Nepal, 2018: Surrogacy services are banned in Nepal”. Disponible en https:// np.usembassy.gov/u-s-citizen-services/child-family-matters/surrogacy-in-nepal/

Valero Heredia, Ana, 2019: "La maternidad subrogada: un asunto de derechos fundamentales", UNED. Teoría y Realidad Constitucional, $\mathrm{N}^{\circ} 43$.

Wikler, Norma J., 1986: "Society's Response to the New Reproductive Technologies: The Feminist Perspectives", Southern California Law Review, Vol. 59, № 5. 
